\title{
Natural Formulations Addressing Skin Challenges in Baby Care
}

\author{
Giada Maramaldi $^{1 *}$, Martino Meneghin ${ }^{1}$, Giuseppe Antognazza $^{2}$, Michela Pollastri ${ }^{2}$ \\ ${ }^{1}$ Indena, Milano, Italy; ${ }^{2}$ Ellegi Biocosmetica, Assago, Italy. \\ Email: "giada.maramaldi@indena.com,martino.meneghin@indena.com,lg.biocosmetica@tiscalinet.it
}

Received November $7^{\text {th }}, 2012$; revised December $9^{\text {th }}, 2012$; accepted December $16^{\text {th }}, 2012$

\begin{abstract}
Addressing skin challenges in baby care formulations is quite a challenge itself, as the formulation not only needs to be active, but it should also meet specific requirements in terms of ingredients, agreability and rational of the formulation design. Object of this study was the realization of a few different cosmetic formulations blending soundly documented natural active ingredients in systems matching the most common requirements of the baby care products. Additionaly, compatibility evidences of the employed natural actives within the tested systems have also been observed. We are therefore presenting the formulation design of four different formulations for baby care: a baby wash, an immediate rash recovery, an after sun emulsion and a calming and soothing product, each one described for the rational of the actives it contains and provided with a detailed formulation design. Specific attention will be directed to those ingredients for which clinical data supporting the expected skin benefits are available.
\end{abstract}

Keywords: Baby Care; Lenitive, Soothing; Natural Active Ingredients; Dermocosmetics

\section{Introduction}

Soothing means having a calming, assuaging or relieving effect. When the word is referred to the skin, it normally indicates providing a relief from skin discomfort of various origins.

Soothing active ingredients may be also defined as calming, emollient and lenitive, and the botanical kingdom is a rich source of biologically active compounds endowed with soothing properties. Several botanical active ingredients have in fact been reported effective in addressing, by various mechanisms of action, the physiological functions our organism uses to maintain a healthy status and skin appearance and counteract skin discomfort. The challenges eventually occurring on the skin may be managed by several soothing actives that modulate the inflammation response.

On a different page, a second target of active soothing ingredients is restructuring the skin whenever the damage has affected the superficial or the deep layers of the epidermis.

Finally a third yet quite relevant target is addressing the sensorial symptoms that skin discomfort carries along, like itch and dryness.

Combinations of natural active compounds endowed with the above mentioned properties are present in all

"Corresponding author. displayed formulations.

Only after all of these particular formulation issues have been considered can a truly quality product be effective in terms of efficacy and performance.

\section{Formulation Designs and Formulations}

\subsection{Immediate Rash Recovery-Formulation ref. \#08}

Aim of this formulation (Immediate Rash recovery, Table 1) model was realizing a rich formulation with a natural approach for the treatment of mild irritations. The formulation is designed according to a "green concept" containing active ingredients endowed with remarkable soothing properties able to achieve a fast recovery of the outmost level of the skin.

PEG-free, paraben-free and silicon-free, this formulation provides a good sensory feeling with excellent body and very mild properties.

\subsubsection{Manufacturing Procedure-ref. \#8}

Phase A was pre-dispersed at $55^{\circ} \mathrm{C}$ and Xanthan gum was added. The mixture was stirred for $30 \mathrm{~min}$. Phase $\mathrm{B}$ was weighed and heated at $75^{\circ} \mathrm{C}$, then phase $\mathrm{A}$ was added at $80^{\circ} \mathrm{C}$. Once the emulsion was formed, it was slightly cooled down to $70^{\circ} \mathrm{C}$ and at this point phase $\mathrm{C}$ was added under homomixing and stirring. 
Table 1. Immediate rash recovery (8).

\begin{tabular}{|c|c|c|c|}
\hline Phase & Ingredient & \% Weight & Characteristics \\
\hline \multirow{5}{*}{ A } & Aqua & 65.00 & \\
\hline & Glycerin & 2.00 & Emollient, skin conditioning \\
\hline & Aqua, sodium phytate & 0.10 & Natural derived \\
\hline & Lecithin, olibanum extract, microcrystalline cellulose & 1.00 & $\begin{array}{l}\text { Natural derived, see detailed } \\
\text { clinical results }\end{array}$ \\
\hline & Xanthan gum & 0.15 & Natural thickener \\
\hline \multirow{12}{*}{$\mathrm{B}$} & Cetearyl alcohol/cetearyl glucoside & 5.00 & Vegetable derived emulsifier \\
\hline & Cera alba (beeswax) & 1.30 & Emollient, skin conditioning \\
\hline & Hydrogenated olive oil decyl esters & 1.20 & Emollient, skin conditioning \\
\hline & Butyrospermum parkii (shea) butter & 2.00 & Emollient, skin conditioning \\
\hline & Prunus amygdalus dulcis (sweet almond) oil & 4.00 & Emollient, skin conditioning \\
\hline & Caprylic/capric triglyceride & 4.00 & Emollient \\
\hline & Oryza sativa (rice) bran oil & 2.00 & Emollient, skin conditioning \\
\hline & Squalane & 6.00 & Emollient, skin conditioning \\
\hline & Vaccinium myrtillus seed oil & 2.00 & $\begin{array}{l}\text { Natural derived, see detailed } \\
\text { clinical results }\end{array}$ \\
\hline & Oleyl alcohol, zanthoxylum bungeanum fruit extract & 0.50 & $\begin{array}{l}\text { Natural derived, see detailed } \\
\text { clinical results }\end{array}$ \\
\hline & Lecithin, tocopherol, ascorbyl palmitate, citric acid & 1.00 & Stabilizer \\
\hline & Tocopheryl acetate & 0.45 & Antioxidant \\
\hline $\mathrm{C}$ & Zinc oxide & 1.50 & Emollient, skin conditioning \\
\hline $\mathrm{D}$ & Aqua, dehydroacetic acid, benzyl alcohol & 0.80 & Preservative \\
\hline
\end{tabular}

At $45^{\circ} \mathrm{C}$ phase $\mathrm{D}$ was added and the final formulation was finally completely cooled down.

\subsubsection{Formulation Characteristics-ref. \#8}

This formulation has a quite high content of naturally derived ingredients, ranging from natural oils to shea butter. The high emolliency of this formulation is provided by a high content of lipidic phase and emollients in general including Zinc Oxide, for instance.

No questioned preservatives or silicons are present, allowing the formulation a green claim. The formulation is also fragrance-free, although a pleasant, perceivable note is due to the content of Zanthoxylum bungeanum Fruit Extract (Zanthalene ${ }^{\circledR}$, by Indena, Italy), which adds a characteristic fragrance to perfume free formulations. The Olibanum extract also contributes to the peculiar note of this formulation.

The formulations, according to final users, provide an immediate soothing activity. On the other hand, it has to be noted that no incompatibilities have been observed among such high load of natural ingredients.

\subsubsection{Rational-Natural Extracts—ref. \#8}

The formulation contains a combination of three different botanical extracts, all standardized and soundly clinically validated.

Olibanum extract: olibanum, also known as Boswellia serrata, is here in the form of a Phytosome ${ }^{\circledR}$, a specific delivery system aiming at improving the bioavailability of selected compounds as polyphenols and triterpenes [1]. Boswellic acids are the anti-inflammatory compounds present in boswellia resin [2], and in the form of this delivery system (Bosexil $^{\mathrm{TM}}$ by Indena, Italy) they have shown a good soothing activity on challenged skin damaged by eczema or psoriasis [3].

Vaccinium myrtillus seed extract: the small seed of bilberry (the wild blueberry, Vaccinium myrtillus) is known to represent a good source of omega- 6 and omega- 3 polyunsaturated fatty acids [4]. The lipids are extracted by $\mathrm{CO}_{2}$ in an ecocert validated process producing an extremely emollient bilberry seed oil (Omegablue ${ }^{\circledR}$, by Indena, Italy) which has been clinically tested as well. Two separate clinical trials have demonstrated the skin barrier restruc- 
turant properties of the bilberry seed oil versus a placebo formulation containing the same lipidic amount $[3,5]$.

Zanthoxylum bungeanum fruit extract: Zanthoxylum bungeanum, also known as Sichuan pepper, is a Chinese spice much employed in Asian gastronomy to reduce the spiciness of hot foods [5]. It contains some alkylamides, among which the most abundant compound is alphahydroxy-sanshool, which interact with temperature and pressure receptors and reduce the skin discomfort and itch sensations [6]. Several clinical evaluations on itch of various origins have confirmed its efficacy [7].

\subsection{Baby Wash-Formulation ref. \#21}

Aim of this formulation (Baby wash, Table 2) design was to obtain a gentle body wash cleanser specifically targeted at children with quite severe disruption of the hydro-lipidic barrier leading to a marked dehydrated skin condition.

The formulation that was obtained is a combination of an oil in water emulsion which is added to a mixture of surfactants, specifically selected as the mildest and least aggressive.

Ideally this formulation is recommended for the daily care of delicate or sensitive skin.

The cleansing portion of the formulation has emollient and soothing properties, cleanses the baby's skin, keeping it soft and protected, while respecting its natural physiological balance.

\subsubsection{Manufacturing Procedure-ref. \#21}

Phase B was weighed and homomixed at $70^{\circ} \mathrm{C}$ for $20 \mathrm{~min}$. Phase $\mathrm{C}$ was weighed, heated at $70^{\circ} \mathrm{C}$, added to phase $\mathrm{B}$ and the blend was homomixed for $5 \mathrm{~min}$, to allow emulsion formation and the emulsion was then slowly cooled down under stirring.

The emulsion of $\mathrm{B}+\mathrm{C}$ phases was then added to phase A (pre-weighed) while stirring.

At $45^{\circ} \mathrm{C}$ phase $\mathrm{D}$ and then phase $\mathrm{E}$ were added.

\subsubsection{Formulation Characteristics-ref. \#21}

This formulation has been specifically designed for containing a surfactant's phase with the mildest and least aggressive compounds. Once again it is a fragrance free formulation, being the pleasant perceivable note given by the content of Zanthoxylum bungeanum Fruit Extract. The formulation is the combination of a hydrolized protein derivative containing phase (B) blended with a tamarind seed polysaccharide, this providing excellent soothing and moisturizing effect. The combination with a high molecular weight silicon ultimately gives the formulation a silky film forming effect.

The result obtained with this formulation is a fragrance free, low foaming mild and gentle cleanser endowed with an immediate moisturizing effect, recommended even for the driest and most sensitive skin types.

Full compatibility between the emulsion and the surfactant system was observed.

Table 2. Baby wash (21).

\begin{tabular}{|c|c|c|c|}
\hline Phase & Ingredient & \% Weight & Characteristics \\
\hline \multirow{4}{*}{ A } & Sodium coceth sulfate & 27.00 & Anionic surfactant \\
\hline & Cocoamido cocoyl hydrolized wheat protein & 2.00 & Natural origin \\
\hline & Caprylyl capryl glucoside & 1.00 & Extremely mild surfactant \\
\hline & Cocoamide dea & 2.00 & Non-ionic surfactant \\
\hline \multirow{4}{*}{$\mathrm{B}$} & Aqua & 58.40 & \\
\hline & Tamarind seed polysaccharide & 0.70 & $\begin{array}{l}\text { Natural derived, see detailed clinical } \\
\text { results }\end{array}$ \\
\hline & Hydrolized wheat protein & 0.50 & Emollient \\
\hline & Hydrolized rice protein & 0.10 & Emollient \\
\hline \multirow{3}{*}{$\mathrm{C}$} & Glyceryl monostearate & 2.00 & Emulsifier \\
\hline & Oleyl alcohol/zanthoxylum bungeanum fruit extract & 0.50 & $\begin{array}{l}\text { Natural derived, see detailed clinical } \\
\text { results }\end{array}$ \\
\hline & Ascorbyl palmitate & 0.10 & Antioxidant \\
\hline $\mathrm{D}$ & Aqua, dehydroacetic acid, benzyl alcohol & 0.80 & Preservative \\
\hline $\mathrm{E}$ & $\begin{array}{l}\text { Divinyldimethicone, dimethicone copolymer and c12-13 } \\
\text { pareth-23 and c12-13 pareth-3 }\end{array}$ & 3.00 & $\begin{array}{l}\text { Silicon derived high molecolar } \\
\text { weight polymer with film forming } \\
\text { properties }\end{array}$ \\
\hline
\end{tabular}




\subsubsection{Rational-Natural Extracts-ref. \#21}

The formulation contains a combination of two different botanical extracts, all standardized and soundly clinically validated:

Tamarind seed polysaccharide: the seed of tamarind, also known as Tamarindus indica, contains several polysaccharides among which the most abundant is xyloglucan [8]. Xyloglucan, when added to cosmetic formulations, has shown remarkable properties in terms of improving skin moist, skin elasticity, skin density and filaggrin expression (being filaggrin closely associated to skin homeostatic balance) [9].

Zanthoxylum bungeanum fruit extract: see efficacy description above.

\subsection{After Sun Relief-Formulation ref. \#18}

Aim of this formulation (after sun relief, Table 3) was the realization of a sprayable emulsion characterized by low viscosity yet a good spreadability, ideal for after sun application targeting local redness.

\subsubsection{Manufacturing Procedure—ref. \#18}

Phase A was weighed and the polymer was predispersed. All components of phase B were separately weighed and stirred in water at $65^{\circ} \mathrm{C}$. Phase $\mathrm{B}$ was added to phase $\mathrm{A}$ while homomixing and stirring. The blend was heated at $70^{\circ} \mathrm{C}$ and phase $\mathrm{C}$ was added while homomixing for 5 min. While cooling down, phase D was added at a temperature of $50^{\circ} \mathrm{C}$. Phase E was separately prepared by predispersing Tamarind seed Polysaccharide in water at $45^{\circ} \mathrm{C}$ and stirring for $30 \mathrm{~min}$. After that, glycerine and phenoxyethanol were added to phase E.

Phase E was then added to the emulsion, which was then cooled down slowly and at $40^{\circ}$ phase $\mathrm{F}$ was added while homomixing.

\subsubsection{Formulation Characteristics-ref. \#18}

This formulation was specifically designed to be a low viscosity emulsion to be sprayed by the means of a hairless pump. The sensorial characteristics describe a light, fresh and easily spreadable emulsion.

The formulation is once again fragrance free, being the pleasant perceivable and slightly spicy note provided by the presence of Zanthoxylum bungeanum and of Olibanum extracts.

Combining light esters with low amounts of silicons (dimethicone) provides a less greasy effect and improves the skin spreadability; it also theorically promotes the delivery of active ingredients.

No incompatibilities were observed between the natural and the synthetic ingredients.

Table 3. After sun relief (18).

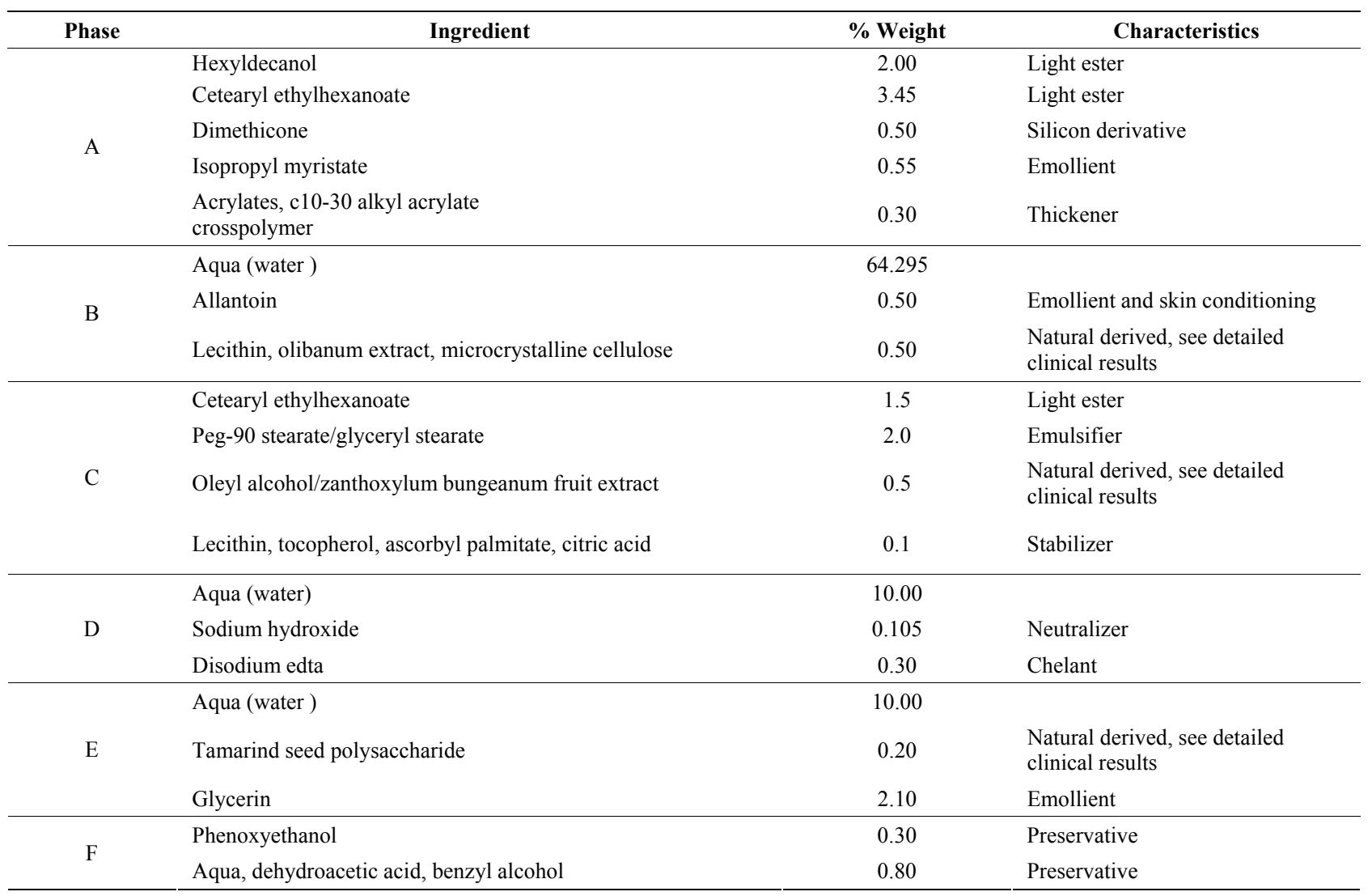


Table 4. Calming and soothing emulsion (17).

\begin{tabular}{|c|c|c|c|}
\hline Phase & Ingredient & $\%$ Weight & Characteristics \\
\hline \multirow{9}{*}{ A } & Cetyl alcohol, c12-20 acid peg-8 ester & 14.00 & Ester \\
\hline & Peg-90 stearate, glyceryl stearate & 4.00 & Emulsifiers \\
\hline & Simmondsia chinensis (jojoba) oil & 8.10 & Natural derived \\
\hline & Olea europaea (olive) oil unsaponifiables & 2.20 & Natural derived \\
\hline & Dimethicone & 0.70 & Silicon \\
\hline & Tocopheryl acetate & 0.50 & Antioxidant \\
\hline & Lecithin, tocopherol, ascorbyl palmitate, citric acid & 0.10 & Stabilizer \\
\hline & Oleyl alcohol, zanthoxylum bungeanum fruit extract & 0.50 & $\begin{array}{l}\text { Natural derived, see detailed } \\
\text { clinical results }\end{array}$ \\
\hline & Esculin & 0.50 & $\begin{array}{l}\text { Natural derived, see detailed } \\
\text { clinical results }\end{array}$ \\
\hline \multirow{8}{*}{ B } & Aqua (water) & 46.700 & \\
\hline & Glycerin & 4.00 & Emollient, skin conditioning \\
\hline & Allantoin & 0.50 & Emollient, skin conditioning \\
\hline & Disodium edta & 0.300 & Chelating \\
\hline & Hydrolized wheat protein & 0.60 & Emollient, skin conditioning \\
\hline & Potassium sorbate & 0.10 & Preservative \\
\hline & Phenoxyethanol & 0.30 & Preservative \\
\hline & Lecithin, olibanum extract, microcrystalline cellulose & 0.50 & $\begin{array}{l}\text { Natural derived, see detailed } \\
\text { clinical results }\end{array}$ \\
\hline \multirow{3}{*}{$\mathrm{C}$} & Aqua(water) & 3.00 & \\
\hline & Imidazolidynil urea & 0.30 & Preservative \\
\hline & $\begin{array}{l}\text { Glucose, sorbitol, urea, sodium pca, glycine/lactic acid, } \\
\text { panthenol } \\
\text { Hydrolyzed wheat protein, sodium glutamate }\end{array}$ & 2.00 & Emollient, skin conditioning \\
\hline \multirow{4}{*}{$\mathrm{D}$} & Aqua (water) & 10.00 & \\
\hline & Sodium hyaluronate & 0.10 & Stabilizer \\
\hline & Aqua, dehydroacetic acid, benzyl alcohol & 0.70 & Preservative \\
\hline & Methylenebisbenzotriazolyl tetramethylbutylphenol & 0.30 & Filter \\
\hline
\end{tabular}

\subsubsection{Rational-Natural Extracts-ref. \#18}

The formulation contains a combination of three different botanical extracts, all standardized and soundly clinically validated:

Tamarind Seed Polysaccharide: see efficacy description above.

Zanthoxylum bungeanum fruit extract: see efficacy description above.

Olibanum extract: see efficacy description above.

\subsection{Calming and Soothing Emulsion- Formulation ref. \#17}

Aim of this formulation (calming and soothing emulsion,
Table 4) was the realization of a smooth face cream with an optimized sensory profile. This formulation has been specifically designed to sooth skin redness resulting from dryness and external factors such us pollution or UV rays.

\subsubsection{Manufacturing Procedure-ref. \#17}

Phase A was weighed, homomixed and heated up to $75^{\circ} \mathrm{C}$. Phase $\mathrm{B}$ was separately weighed, heated at $75^{\circ} \mathrm{C}$ and then added to phase $\mathrm{A}$ while homomixing under vacuum.

Phase $\mathrm{C}$ components had been predispersed, heated at $50^{\circ} \mathrm{C}$ and the added to the newly formed emulsion. 
A sodium hyaluronate predispersion was separately prepared, and then all other phase D components have been added. Phase $\mathrm{D}$ was added to the emulsion during the cool down step at a temperature of $45^{\circ} \mathrm{C}$.

\subsubsection{Formulation Characteristics-ref. \#17}

This formulation is a rich pot cream specifically formulated with highly skin conditioning emollients and natural oils as Jojoba oil and Olive Oil Unsaponifiable.

The combination with oily moisturizers and soothing ingredients such as Zanthoxylum bungeanum and of Olibanum extract help to create an artificial barrier preventing itching and skin discomfort.

As all the previously described, this is a fragrance-free formulation as well with a perceivable note provided by the olibanum extract. Besides the natural oils and the skin conditioners, it also contains natural active ingredients of capillary protection, soothing and assuaging properties.

\subsubsection{Rational-Natural Extracts-ref. \#17}

The formulation contains a combination of two different botanical extracts, all standardized and soundly clinically validated:

Esculin: Esculin is a pure molecule extracted from the bark of the branches of Horse Chestnut, also known as Aesculus hippocastanum. Esculin has shown a capillary protective action in a couple of clinical trials so far, where it was shown to reduce capillary fragility and exert anti-inflammatory properties [10].

Zanthoxylum bungeanum fruit extract: see efficacy description above.

Olibanum resin extract: see efficacy description above.

\section{Discussion}

The above-mentioned formulations are provided as the results of a detailed systematic study aimed at verifying the compatibilities of natural active ingredients in different formulation models.

Natural ingredients, in fact, due to the variability of the natural material, may encounter a broad variability in terms of safety and efficacy performances. Only when a strict standardization is in place the natural material is highly reproducible. Standardization of a botanical extract means that all steps form the very beginning of the selection of the most appropriate variety of the botanical ingredient are closely defined and controlled, so that the variability of the active compounds, as well as the fingerprint of the phytocomplex itself, is the least variable.

The described formulations contain botanical active ingredients all aiming at a soothing, lenitive and calming effect. They have been combined in different systems and no crucial issues have been observed.

\section{REFERENCES}

[1] A. Semalty, M. Semalty, M. S. M. Rawat and F. Franceschi, "Supramolecular Phospholipids Polyphenolics Interactions: The PHYTOSOME ${ }^{\circledR}$ Strategy to Improve the Bioavailability of Phytochemicals," Fitoterapia, Vol. 81, No. 2, 2010, pp. 306-314. doi:10.1016/j.fitote.2009.11.001

[2] S. Singh, A. Khajuriaa, S. C. Tanejaa, R. K. Johria, J. Singha and G. N. Qazia, "Boswellic Acids: A Leukotriene Inhibitor Also Effective through Topical Application in Inflammatory Disorders," Phytomedicine, Vol. 15, No. 6-7, 2008, pp. 400-407. doi:10.1016/j.phymed.2007.11.019

[3] Indena, "Velleja Research," 2012.

[4] E. Bere, "Wild Berries: A Good Source of Omega-3," European Journal of Clinical Nutrition, Vol. 61, No. 3, 2007, pp. 431-433. doi:10.1038/sj.ejen.1602512

[5] AAVV, "Omegablue ${ }^{\circledR}$, Olio di Semi di Mirtillo con Proprietà Restitutive," Cosmetic Technology, Vol. 13, No. 6, 2010, pp. 66-67.

[6] R. C. Lennertz, M. Tsunozaki, D. M. Bautista and C. L. Stucky, "Physiological Basis of Tingling Parestesia Evoked by Hydroxy Alpha Sanshool," The Journal of Neuroscience, Vol. 30, No. 12, 2010, pp. 4353-4361. doi:10.1523/JNEUROSCI.4666-09.2010

[7] C. Artaria, G. Maramaldi and G. Appendino, "Sichuan Pepper as a Skin Spice," Journal of Applied Cosmetology, Vol. 29, 2011, pp. 87-98.

[8] M. Gidley, et al., "Structure and Solution Properties of Tamarind Seed Polysaccharide," Carbohydrate Research, Vol. 214, No. 2, 1991, pp. 299-314. doi:10.1016/0008-6215(91)80037-N

[9] G. Maramaldi, S. Togni, S. Varesi and A. Giori, "Tamarindus Indica Xiloglucal," Cosmetic Technology, Vol. 14, No. 5, 2011, pp. 17-21.

[10] E. Bombardelli, P. Morazzoni and A. Griffini, “Aesculus hippocastanum L.," Fitoterapia, Vol. 67, No. 6, 1996, pp. 483-511. 